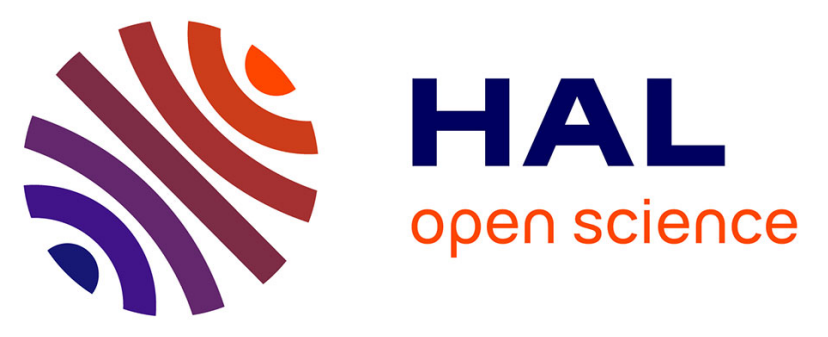

\title{
Perception of Multisensory Wind Representation in Virtual Reality
}

Gabriel Giraldo, Myriam Servières, Guillaume Moreau

\section{To cite this version:}

Gabriel Giraldo, Myriam Servières, Guillaume Moreau. Perception of Multisensory Wind Representation in Virtual Reality. ISMAR 2020 - 19th IEEE International Symposium on Mixed and Augmented Reality, Nov 2020, Recife, Brazil. pp.89-97, 10.1109/ISMAR50242.2020.00024 . hal-02924372

\section{HAL Id: hal-02924372 \\ https://hal.science/hal-02924372}

Submitted on 28 Aug 2020

HAL is a multi-disciplinary open access archive for the deposit and dissemination of scientific research documents, whether they are published or not. The documents may come from teaching and research institutions in France or abroad, or from public or private research centers.
L'archive ouverte pluridisciplinaire HAL, est destinée au dépôt et à la diffusion de documents scientifiques de niveau recherche, publiés ou non, émanant des établissements d'enseignement et de recherche français ou étrangers, des laboratoires publics ou privés.

\section{(1) (1) $\$$}

Distributed under a Creative Commons Attribution - NonCommercial - NoDerivatives 44.0 


\title{
Perception of Multisensory Wind Representation in Virtual Reality
}

\author{
Gabriel Giraldo* Myriam Servières Guillaume Moreau \\ Ecole Centrale de Nantes, AAU CNRS UMR 1563, Nantes, France
}

\begin{abstract}
The set of physical and sensitive phenomena that interacts with the urban morphology acts on the resulting perception from the users of a place. Its study and representation provides elements beyond the aesthetics aspects that can allow a better understanding of the space and future urban projects. We aim to analyze the effects of three different wind representations in terms of perception and sense of presence in virtual reality (VR). We focus on the following conditions: $(\mathrm{R})$ reference scene with the audiovisual representation of the mechanical effects of the wind on the elements of the context, (V) reference scene plus the visualization of the wind flow, present (among others) in the architecture field, $(\mathrm{T})$ reference scene plus tactile restitution of wind and eventually $(\mathrm{V}+\mathrm{T})$ assembling all previous conditions. For the experiment, we present to the participants $(\mathrm{R})$, then $(\mathrm{V})$ followed by $(\mathrm{T})$, and finish with $(\mathrm{V}+\mathrm{T}) .37$ participants evaluated 12 different stop points (divided into four routes in the same simulated street), where they had to determine the perceived wind force and direction concerning the four different conditions (each one corresponding to one route). At the end of each route, participants evaluated their sense of presence in the VR scene. Our analysis showed significant effects of tactile restitution over the visual effects used in the study, both for understanding wind properties and for increasing the sense of presence in the VR scene. In terms of wind direction, $(\mathrm{T})$ reduced the estimation error by $27 \%$ compared to $(\mathrm{V})$. Concerning wind force, the reduction was $9.8 \%$. As far as presence was concerned, $(\mathrm{T})$ increased the sense of presence by $12.2 \%$ compared to $(\mathrm{V})$.
\end{abstract}

Keywords: Wind Perception, Wind Representation, Virtual Reality, User Experiment, Sense of Presence, CFD Visualization, Urban 3D model.

Index Terms: Human-centered computing-Human computer interaction (HCI) - HCI design and evaluation methods-User studies.

\section{INTRODUCTION}

Virtual reality (VR) has demonstrated its potential in the architecture field by, among others, its ability to allow immersion of users into a 3D model [31]. Beyond aesthetic aspects, VR has the potential to represent both physical and sensitive phenomena; in previous studies, Vigier et al. [43] have shown how to suggest different climatic behaviors using color and contrast changes. Tahrani and Moreau [40] studied the relationship between daylight, space, and human cognition in a VR scene compared to the real world. In this study, we are interested in the representation of the wind which plays an important role in terms of health, thermal, and mechanical comfort of users of the urban space [15].

Indeed the wind can modify the user's perception of a space and how it will be used in real life. However, it is barely present in the project's representations, and when it is, it is only represented by visual aspects and quantitative values, leaving aside the sensitive properties of the phenomenon. Berger and Cristie [3] explored a

*surname.lastname@crenau.archi.fr computational fluid dynamics (CFD) representation in VR to bridge the gap between architects and engineers in this context and to allow collaborative work around it. They presented an animation showing a way to express the properties of wind direction and force.

In the current study, we aim at comparing different VR representations of the wind to identify their incidence on the understanding of wind properties and the sense of presence in a VR scene. Four different wind representations were presented to participants (i) the audiovisual representation of the mechanical effects of wind on the context elements, (ii) the addition of the wind flow visualization to (i), (iii) the addition of the tactile restitution to (i), and finally, (iv) all these representations together (See in Sect. 3.3)).

The user experiment compares these different types of representation to communicate and evaluate the understanding of wind properties coming from a CFD simulation and its effects on the sense of presence in a VR scene. We explore a new way to communicate those CFD simulation results of an urban project to suggest the wind presence and its incidence on the context. Results show a higher efficiency in understanding wind properties using a condition with tactile restitution. In the same way. The tactile restitution was also the condition that most increased the participants' sense of presence.

The remainder of this paper is structured as follows: in Sect. 2 we present the previous works about wind representation in different domains and their influence in our prototype. Sect. 3 describes our user study, its hypotheses, and the prototype we have designed. Results are presented in Sect. 4 and discussed in Sect. 5.

\section{Related Work}

The direct effects of wind can be felt by various senses: it provides audio, tactile, and thermal feedback to the human body. Its effects can also be indirect, and, in this case, also visual: the wind makes the leaves of the trees move, which is of course visible. Therefore, we decided not to limit our literature review to direct stimuli, but also to consider such indirect stimuli. We also studied the world of sensory 'replacement', i.e. suggestions.

In this section, we present the three main groups of wind representations that we have found in the literature and that are the basis of our prototype. We first present the wind visualizations used in the field of architecture and engineering, whose main feature is to make the wind flow path visible. As this may be barely legible for the general audience, we then review the wind representations in artistic works, where the wind presence is shown by its mechanical effects on the context elements. Finally, we end with the first attempts of wind tactile rendering in virtual reality scenes.

\subsection{Wind Visualization for Architecture and Engineering}

Representations of wind in architecture, engineering, and urban design projects are mainly visual ones. They help designers to materialize this invisible phenomenon in order to study it, analyze it, measure it, and use it for decision-making. These representations are mainly based on suggestive sketches that allow to understand the basic properties of wind and on simulation results that provide more quantitative information. Both are present in the elaboration phase of the projects and allow the discussion between professionals [7,22].

At the time of project communication, we find those representations again. On one side, sketches such as the graphic representations shown by Gandemer [15], are used to explain the wind behavior in 
relation to buildings morphology. Different effects resulting from the interaction of wind and urban shapes (e.g., Venturi effect, row effect, corners effect, etc.) are shown by sketches, translating the wind flow behavior into arrows (see in Fig. 1). The same principle is found in the Lamberts et al. [22] publication, which has the purpose of telling how the wind interacts with the environment. The authors opt for a handmade, cartoon-like representation to guide and introduce the reader intuitively to these concepts. Similarly, Bustamante et al. [7] use this kind of representation to make visible existing issues that are the result of the interaction between the physical phenomenon and the built environment. They explain how the geographical conditions of the city, and its relation with the urban shapes, produce discomfort to pedestrians walking on the street. These representations make it possible to understand the phenomenon behavior and its properties, such as its dominant direction or an idea of its intensity.

On the other side, we find the wind simulations. They allow us to analyze and quantify wind dynamics to enrich the project according to morphological, conceptual, or structural choices. Like the sketches, the main goal in these representations is to make visible the wind flow behavior. As seen in the extensive reviews of flow visualization provided by Edmunds et al [11], McLoughlin et al. [27] and Salzbrunn et al. [37], there are different ways to communicate the wind properties resulting from a CFD simulation. Post et al. [34] identify four main groups (i) direct visualization (raw data are communicated in the most direct way, showing flow properties by colors or arrows from vectors), (ii) texture-based visualization (noise textures are added to the flow visualization in order to show density or displacement), (iii) geometric visualization (add geometric objects like streamlines, streaklines, pathlines, and isosurfaces, showing the flow trajectory and a spatial definition) and (iv) featurebased visualization (is a synthesis and assembly of data with similar characteristics that shows only the information that is of interest for the researcher). Salzbrunn et al. [37] adds a fifth group, the (v) partition-based visualization (gives an overall result of the behavior of the fluid and its environment). According to Laramee et al. [23] (i) is intuitive and easy to use. However, it is difficult to apply in a $3 \mathrm{D}$ representation, unlike (iii). We share the idea that geometric visualizations (both by lines and by isosurfaces) are adapted to the 3D representations. However, while isosurfaces can be the most useful representation in an overall view, from an immersive point of view, in an urban scene, they may be confusing (see Fig. 2, left image). We believe that streamlines can be better adapted to the immersive point of view used in VR (See Fig. 2, right image). Brambilla et al. [6] focuses on illustrative flow visualization, where scientific representations benefit from inspiration from traditional handmade sketches. Representations are classified based on the types of data the user wants to see on the rendering, (i) raw data, (ii) integral structures, and (iii) flow features.

We then find a common interest in both CFD results visualization and suggestive sketches: the visibility of the wind flow, i.e. the materialization of this invisible phenomenon. We assume a special link exists between sketches and geometric visualization because both representations seek to visualize and trace the trajectory of an element representing the wind flow.

We aim at exploiting the assets of these visualizations by postprocessing the CFD simulation results and use them in the VR scene.

An example of this post-processing stage is given by Berger and Cristie [3], where a dynamic CFD simulation result was presented in a VR scene. They create an animation of streamlines that enables to see the evolution of wind flow through a 3D model along time. We show another example in a previous research [16], where the CFD simulation result is superimposed onto a photograph of the real space in a video, in order to communicate a temporal evolution of wind flow.
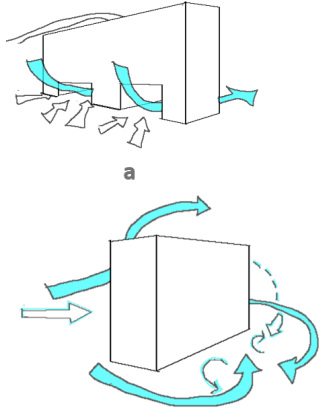

d
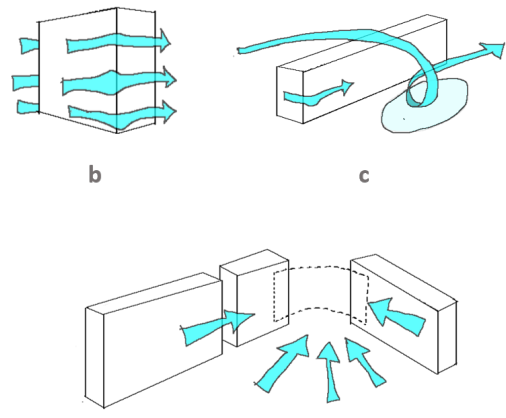

e
Figure 1: Example of wind effects, flow visualization (a) effect of slots under a building, (b) effect of corners, (c) row effect (d) wake effect and (e) Venturi effect, based on Gandemer's [15] sketches.
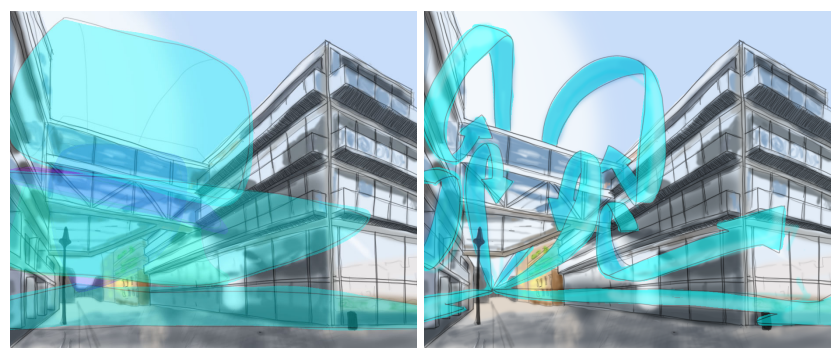

Figure 2: Sketch of a wind visualization in an immersive point of view. Left: Isosurfaces representation; Right: Streamlines representation.

\subsection{Wind Representations in Art and Computer Graph- ics}

In artistic representations, wind expressions are mainly linked to the mechanical effects of the wind on the context elements.

In static images (photographs or paintings), the dynamic nature of the wind can be reproduced by a blur effect on some objects. It provides a displacement notion, e.g. the blurred dry leaves and umbrellas contrast with the sharpness of the buildings in the photograph presented by Blocken et al. [4], where the wind direction can be perceived. Another similar example is found in painting, e.g Barr's painting [2] where the blurred traces dematerialize the edge of the building and communicate the direction of the wind.

In cinema, wind presence is suggested by its influence on the surrounding elements. In the films "Paperman" [19], "The happening" [38] and "The Blue Umbrella" [41], wind presence is evident throughout the development of stories. On the one hand, wind mechanical effects are visible on trees, grass, hair, and characters clothes. On the other hand, the sound effects resulting from the wind blowing play an equally important role.

There are examples in painting, like the "Birth of Venus" by Boticelli [5] and in cinema, like the animated film "Pocahontas" [14] where, in addition to the visual and audio effects, the wind flow itself becomes visible to express its properties and presence (as shown in Sect. 2.1).

This topic has also been addressed in Computer Graphics. Wang et al. [44], show the direction and wind force expressed by the wind influence on the snowfall. Dobashi et al. [10], Max et al. [26] and Vigier et al. [43] suggested the wind properties by cloud motion in a scene. Finally, wind presence is expressed on the green elements, e.g. in grass [1] or trees deformation $[9,35]$. 
To take these works into account, we used an audiovisual suggestion of the mechanical effects of wind on the elements of context to generate our reference scene.

\subsection{Tactile Restitution of the Wind in VR Scenes}

Tactile wind restitution has been used in virtual reality devices by using fans [8, 20, 25, 28,33, 42], compressed air [36], and a scaled wind tunnel [21], detailed below.

Moon and Kim [28] proposed the windcube, a cubic device that surrounds users. The layout of the fans consists of three different levels, where each one of the two lower levels has eight fans and the superior level has four. The fans on the lower levels were placed equidistantly every $45^{\circ}$ in a circle. This device fulfilled its purpose of making it possible to evaluate the sense of presence of people according to the wind. Nevertheless, the authors noted that it could be visually invasive and therefore could distract users, reducing, paradoxically, their sense of presence in the VR scene. This layout of eight fans was reused by Cardin et al. [8] using a head-mounted display (HMD), and Verlinden et al. [42]. Cardin et al. [8] propose a "head-mounted wind" device, which consists of an HMD adapted with eight fans. The device was tested in a flight simulation in order to evaluate the perception of wind direction. In Verlinden et al. [42] research, the application scale of the fan layout used by Moon and Kim [28] was changed: they used eight fans with a $40 \mathrm{~cm}$ diameter placed in an upper structure that had a $4 \mathrm{~m}$ diameter and was $2 \mathrm{~m}$ high. The device was tested in a sailing simulator where the sense of presence was evaluated. Results showed that it was increased in situations where the wind restitution was used. Pluijms et al. [33] used the same device as Verlinden et al. [42], in order to evaluate the perception of wind direction and force between expert sailors, less skilled sailors, and inexperienced controls. Wind direction estimation showed more accurate for expert sailors than for non sailors.

Kojima et al. [20] adapted a helmet with small fans, tubes and an audio speaker, in order to apply the wind directly around the ear, the most sensitive area to the wind. Rietzler et al. [36] developed a pneumatic device that can rotate $270^{\circ}$ around the user's head providing visual and audio content. They noted an increase in the sense of presence and enjoyment in the VR scene using the tactile restitution. They noted a potential problem with the HMD use that masks a part of the face. They suggested adding glasses to the virtual avatar in order to reduce the problem. In the same way, they suggest adding gloves to the character because the wind effect was not present at the hands level. This leads to the conclusion that HMDs are not really suitable for tactile restitution of wind.

Kulkarni et al. [21] use cross-approaches between numerical and physical simulations in order to include the wind behavior in a VR device. Their main goal is to develop a device that uses wind, temperature and odors to increase the number of senses stimulated and thus increase the sense of presence in VR. The air is directed through two side ducts to the oblique screens of the device, which creates a vortex and directs the wind flow towards the user according to its angle of incidence and speed.

Lehmann et al. [25] compared the use of fans that accompany a video game displayed on a screen in two situations. The first one, with two static fans located in front of the user. The second one, with two fans attached to a helmet and located next to the ears. The pre-test showed a tendency that static fans better support the feeling of presence than head-mounted wind. One of the reasons given was the possible vibration and sound produced by the fans in the helmet. However, both situations increased the sense of presence in the virtual reality scene compared to a situation without wind. This result is consistent with other studies [28,36,42].

More recently, Ito et al. [18] produced a demonstration integrating visual, audio and tactile stimuli with a limited number of fans and taking into account cross-modal effects. However, this prototype has not been fully evaluated yet.

Nakano et al. have conducted different studies to find out whether subjects can discriminate wind direction according to the area of the face exposed to the wind [29]. They have compared JNDs (Just Noticeable Differences) values of wind direction discrimination produced by one single fan or a uniform wind produced by a fan array [30] when the wind is blowing on the face. They have found that the JND for a uniform wind was $5.55^{\circ}$ if the subject concentrates and the wind is directly blown on the face with no other stimulus.

\subsection{Summary}

According to the experiments shown by Lehmann et al. [25], Moon and Kim [28], Rietzler et al. [36] and Verlinden et al. [42], the addition of the tactile restitution to an audiovisual representation of the mechanical effects of the wind increases the sense of presence in a VR scene. Wind properties, direction and force, were also tested in VR in order to evaluate the user perception. In the mean time, wind properties are shown in the architecture field by wind flow visualization. This kind of representation is also used in the VR field. However, it has neither been compared to the tactile restitution nor to the representation of the mechanical wind effects. We propose to compare those three kinds of representation (the visualization of the wind flow shown in section Sect. 2.1, the suggestion of the mechanical effects of the wind presented in Sect. 2.2 and the tactile restitution shown in Sect. 2.3), that, to the best of our knowledge, has never been done before. We will study the perception of wind force and direction as well as the sense of presence in a VR scene.

\section{Methods}

In this section, we present our experiment which aimed at comparing different wind representation based on a CFD simulation result, in order to evaluate its incidence on the understanding of wind properties and the sense of presence in a VR scene.

The experiment involved 37 participants (17 women and 20 men), aged from 22 to 61 years (mean=35, SD=9.6). They did not know the purpose of the experiment and were not paid for their participation.

\subsection{Apparatus}

We based our experiment on the layout of the fans used by Pluijms et al. [33] and Verlinden et al. [42]. For the sake of visibility, fans were not placed at eye level. While Verlinden et al. [42] put them over the user's field of view, we decided to put them on the floor (see in Fig. 3 lower picture) as it is easier and the participants will be eager to raising their heads to see the buildings and wind streamlines. We used ProBreeze $51 \mathrm{~cm}$ floor fans located around the participant in a $2.6 \mathrm{~m}$ diameter circle. Participants were located at $1.32 \mathrm{~m}$ of the fans and a $1.5 \mathrm{~m}$ of the screen. The screen dimensions were: $1.80 \mathrm{~m}$ high by $2.40 \mathrm{~m}$ wide, providing a $78^{\circ}$ horizontal field of view (see in Fig. 3 upper image).

We established the communication between the VR scene in Unity3D and the real world, with a VMA101 Velleman card based on an ARDUINO ATmega2560 card. The card received the signal from the VR scene and sent it to a relay module to which the fans were connected. Note that we could only use the three predefined speeds of each fan.

In order to render the wind sound, we used wireless in-ear headphones (Plantronics backbeat go 410). They had a double function: to render the wind sound from the VR scene and to reduce the resulting noises from the fans, the relays, and other equipment in the room with its noise-canceling function. Their size did not prevent wind contact in the ears of the participants.

\subsection{VR Scene}

The entire virtual prototype was based on the CFD results obtained from Houda Belgacem (former Ph.D. student of the laboratory). 
As our goal of this work is to study wind perception and not wind simulation, the CFD model that we used is simply a plausible input and could be replaced by any other simulation result or set of real measurements.

The results were processed with ParaView software, from which we generated two different data sets: (i) a horizontal cut, located at a height of $1.5 \mathrm{~m}$, containing wind direction and force (see Fig. 4), which was used to produce the tactile restitution and the sound spatialization (explained in Sect. 3.2.1), (ii) the layout of the streamlines along the street of interest, containing the same properties in order to produce the wind flow visualization in the VR scene as explained in Sect. 3.2.2 (see Fig. 5). Subsequently, both types of information were exported to comma-separated values (CSV) files and were read in the VR scene. The scene was built using Unity3D v2018.3.9f1 We opted for a non-textured 3D model not to distract participants with those visual aspects, and guide them toward focusing on the wind representation as suggested by Park et al. [32].

In our model, the doors and windows' geometric layout was detailed, and the only colors that stood out were the green of trees and the blue of the sky (see Fig. 7).
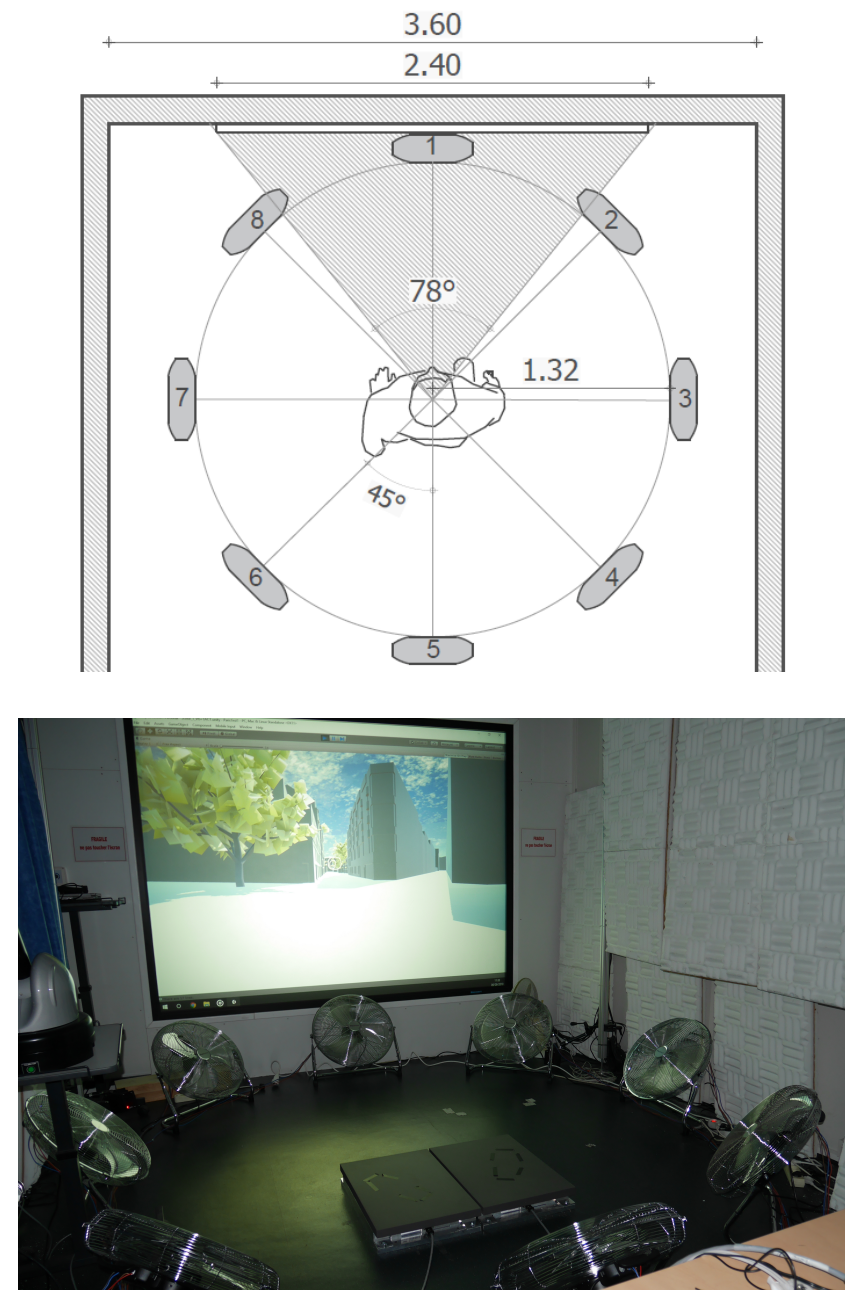

Figure 3: Location of the fans in front of the projection screen. Top image: fan layout around participants, units in meters. Lower picture: photography of the VR room.

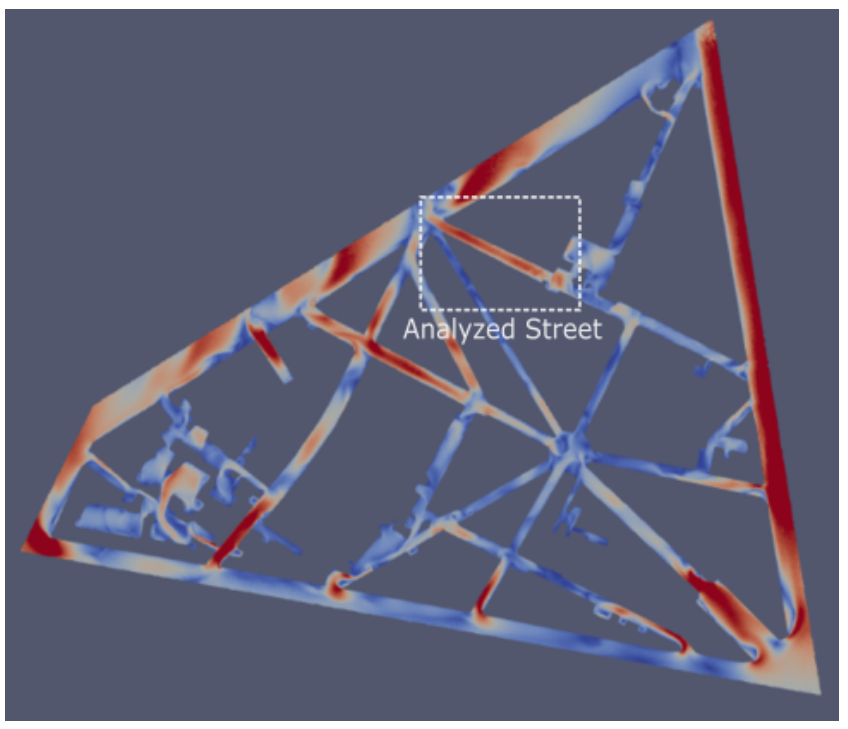

Figure 4: CFD results, horizontal cut of wind speed visualization.

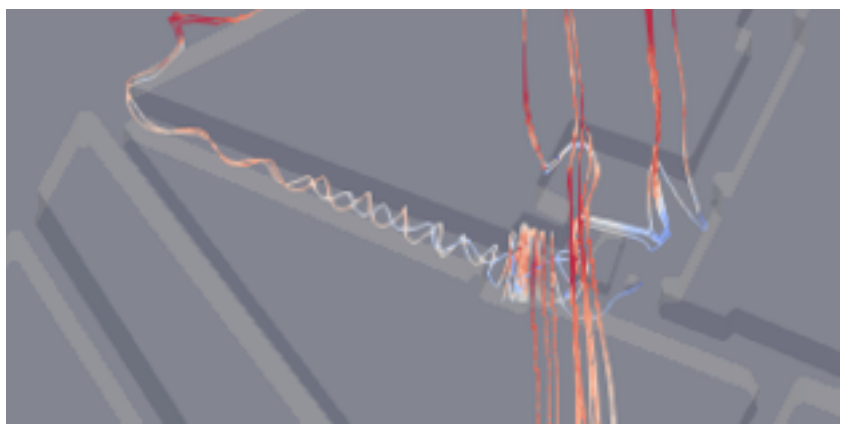

Figure 5: Streamlines of wind velocity visualization in the 3D model of the street of interest.

\subsubsection{Tactile and audio restitution}

The horizontal cut provides a vector field along the studied street: it consists of a file containing a set of points $(x, y, z)$ and their associated wind vector $\left(v_{x}, v_{y}, v_{z}\right)$ with a resolution $<1 \mathrm{~m}$. In order to identify the wind result in the virtual avatar position, we created an octagon around it, with each of its faces matching a fan position in the experimentation room.

All vectors intersecting a face of the octagon (see in Fig. 6, left) are used to compute an average wind vector for a given face. This average vector is orthogonally projected on the fan axis to compute a wind speed for each fan. As each fan has only 4 possible speeds ranging from 0 to maximum speed, a quantification is thus necessary. It is done by assigning the maximum fan speed to the maximum wind speed computed by the simulation and a linear subdivision. However, it has to be noted that we are not seeking accuracy of wind rendering but a mere feeling of various wind speeds as our fans had only 4 possible speed levels. Fans are then activated at the determined speed using the Velleman microcontroller.

The virtual sound source is located at the fans' location. Sound level is determined by estimated wind speed through the fan and then propagated using Unity's standard 3D sound source asset. Again, we are not aiming for full accuracy but are satisfied with a few perceptual levels. 

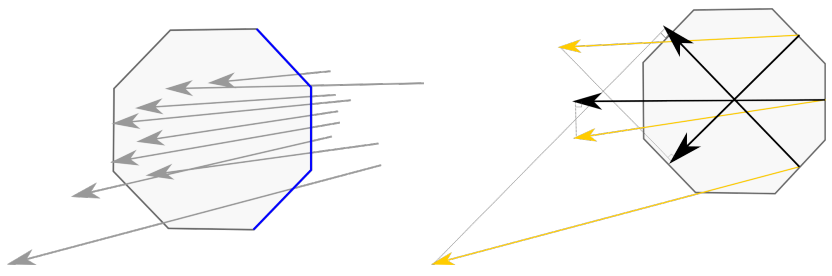

Figure 6: Left: schematic virtual octagon identifying all vectors crossing each surface from outside to inside (the surfaces crossed are indicated in blue). Right: Average vector of wind direction and speed for each fan surface (yellow) and projection on the fan axis (black).

\subsubsection{Visual Wind Flow Representation}

The structuring vectors of the streamlines are coming from the CFD results: streamlines were directly generated by Paraview software (V5.5.0, Stream Tracer function with the following parameters: vectors menu set as velocity, Integration direction: Forward, Integrator type: Runge-Kutta 4-5, Seed Type: High resolution line source, Resolution: 20). The data were exchanged offline between Paraview and Unity software through CSV files. Those files contain for each streamline both the trajectory of seed points though time and the associated wind vector. They were used to create Unity particle paths on which a 3D object was animated at a speed that was proportional to the locally estimated wind speed. This allowed us to create an animation along the streamlines, making visible the wind flow along the streets and visualizing the resulting effect of the interaction between the wind and the built context (see in Fig. 8). As the streamlines exported with Paraview have a limited duration, they were played in loops after a pause to avoid discontinuity in representation.

\subsection{Experimental Design}

The study used a within-subjects design. There was one independent variable, the type of wind representation, with four different levels which were presented to the participants in the following order:

- R: Reference scene composed of an audiovisual representation of the mechanical effects of wind on the elements of context, as found in traditional cinema. The direction and force of the wind were given by the spatialized wind sound. The presence of the wind was also suggested by the movement of tree leaves and clouds (see in Fig. 7). Note that these effects were identical in all scenarios.

- V: Reference scene $(\mathrm{R})+$ the visual representation of the wind flow, as found in the representations used in architecture (see in Fig. 8).

- T: Reference scene $(\mathrm{R})+$ tactile restitution of the wind with digitally controlled fans.

- V+T: Reference scene $(\mathrm{R})+$ visual representation of the wind flow $(\mathrm{V})+$ tactile restitution $(\mathrm{T})$.

The three dependent variables were the perception of wind properties (D1: direction and D2: force), and D3: the sense of presence in the VR scene.

We created four different predefined paths along the same virtual street. We chose to predefine them in order to reduce the time between fan activation and wind perception which was measured and calibrated for the experiment. Each tour had three stop points where participants had to evaluate the perceived direction and force of the wind. The stops points were divided into three different zones to provide similar conditions in terms of built environment. In order to randomize the tours, we used a Latin square with which we obtained

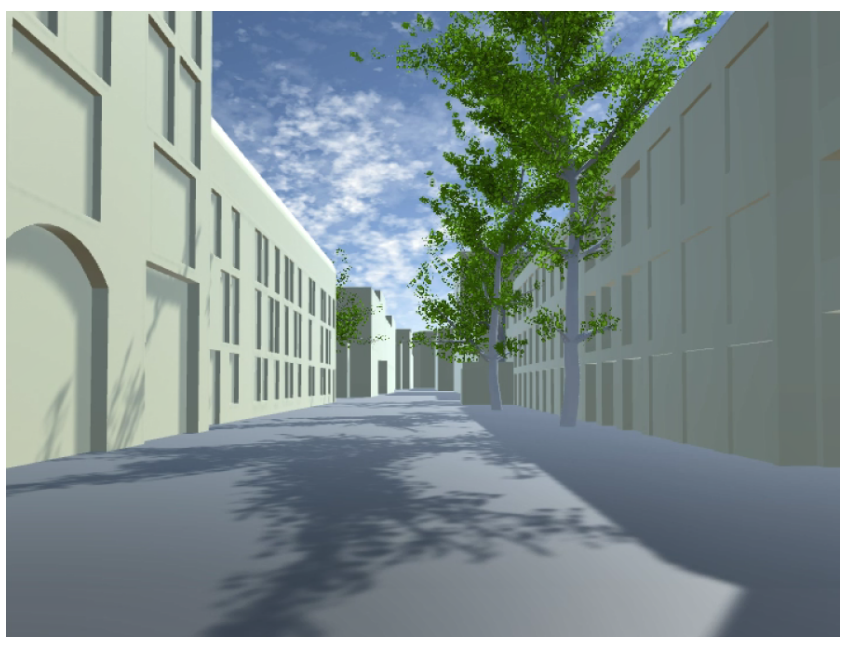

Figure 7: Reference VR scene. First path presented to participants, without visual wind flow effects or tactile stimulation

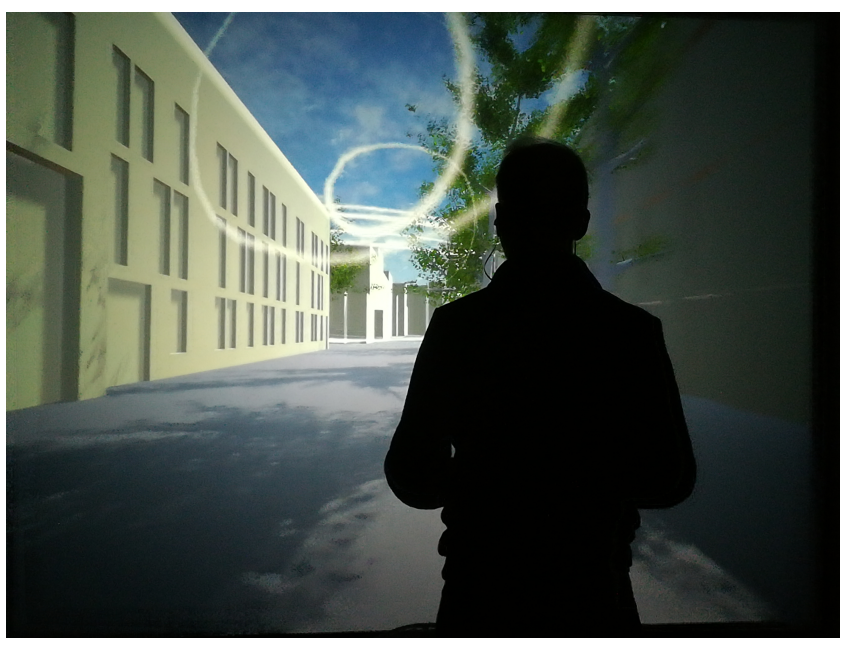

Figure 8: Participant in the VR scene with the visual representation of the wind flow.

four groups of participants. Participants saw the representations in the same order, but they followed different orders of tours depending on their group.

\subsubsection{Hypotheses}

Considering previous research, and based on our experimental design, we made the following hypotheses:

- H1: $\mathrm{V}$ is more accurate for understanding wind direction compared to $\mathbf{T}$. Both in the real world and in virtual reality, a visual preeminence exists: the visual sense supplies much more data to our brain, and at a much greater speed than others senses [17]. By assuming visual preeminence, we believe that the wind flow visualization can bring more accurate information in relation to the wind direction.

- H2: $\mathbf{V}+\mathbf{T}$ is more accurate for understanding wind direction compared to $\mathbf{V}$ and $\mathbf{T}$. Assuming $\mathrm{H} 1$ is correct, visual plus tactile congruence should lead to a better understanding as well. 
- H3: $\mathbf{T}$ is more accurate for understanding wind force compared to $\mathbf{V}$. Because the variations in force produce pressure variations in our body sensors, we assume that the tactile effect could be better than the visual speed variation of the streamlines to perceive the wind force.

- H4: $\mathbf{V}+\mathbf{T}$ is more accurate for understanding wind force compared to $\mathbf{V}$ and $\mathbf{T}$. Again, congruence of senses should lead to better wind perception as $\mathrm{H} 3$.

- H5: $\mathbf{T}$ is more accurate for increasing the sense of presence in the VR scene, compared to V. Previous studies [25, 28, 36, 42] have shown that tactile effects increase the sense of presence in a VR scene compared to an audiovisual representation. We assume the wind flow visualization $(\mathbf{V})$ in our study will be consistent with those previous works.

- H6: $\mathbf{T}$ is more accurate for increasing the sense of presence in the VR scene, compared to $\mathbf{V + T}$. In this case, we do not think the addition of the two variables could be better, because the wind flow visualization does not correspond to reality, we don't see the phenomenon by itself in the real world, we just feel it.

\subsubsection{Procedure}

Each participant came to the virtual reality room where the procedure was explained. He or she had to take four different virtual paths in the same street. Each path was characterized by a type of representation and was presented to participants according to the order given above. Before starting, participants were given a tablet, the noise-canceling headphones and asked for their height in order to adjust the camera point of view. Then, they were located in front of the screen and at the center of the group of fans. A reference wind value was played depending on the condition in order to let the participants identify the maximal values of wind force at sound and tactile level.

The tablet had an application that indicated the number of the stop to evaluate and allowed the participants to specify the wind direction in a circle and the wind force in a slider from 0 (corresponding to no wind) to 10 (corresponding to a reference value given in audio and tactile restitution that was shown to the subjects before the experiment) (see Fig. 9).

At the end of each route, the participants had to come to the desk in order to evaluate their sense of presence in the VR scene with a Slater-Usoh-Steed (SUS) questionnaire [39]. They then restarted the next route with another type of representation.

Each route was 2 minutes long, and the whole experiment lasted between 20 and $25 \mathrm{~min}$ for every participant. None reported any kind of sickness.

\section{Results}

In this section, we present the descriptive and inferential statistical analyses of the perception of the wind properties (direction and force), and of the sense of presence in the VR scene.

\subsection{Statistical Analyses}

The analysis considered the difference between the initial simulated parameters of the wind in the scene (rendered in various experimental conditions) and the user answers for the wind properties, and the sense of presence.

A square root transformation was applied to the data in order to normalize the absolute values of the answers. The resulting values, in relation to wind properties (direction and force) and presence, were analyzed using a multilevel linear model, which is a regression that considers dependency in data [12]. The model includes the variable to be explained (direction difference, force difference or presence), the explanatory variable (the type of representation) and the participant factor as a random effect. The assumption of

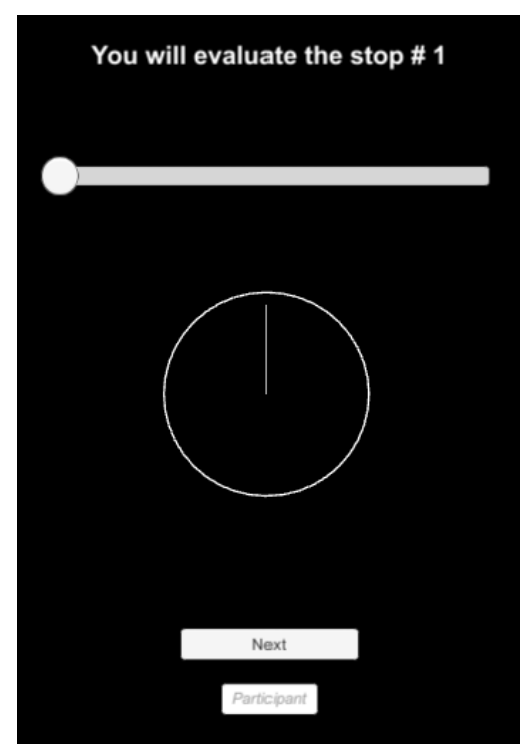

Figure 9: Screenshot of the evaluation app (direction and force).

normality was tested with the Shapiro-Wilk test at the 5\% level. Tukey's post-hoc test was performed to compare all the conditions of the categorical variable representation. The statistical analysis was conducted using the $\mathrm{R}$ software, with the function lme of the nlme package. The glht (multcomp package) function was used for pairwise comparisons and the shapiro.test (stats package) function for the assumption of normality.

\subsection{Wind Direction}

The experimental condition had a significant effect on the estimation of wind direction, $\chi^{2}(3)=66.87, p<0.0001$. Orthogonal contrast revealed that direction error (difference between the expected response and the value given by the participants) was significantly reduced for $\mathbf{T}$ compared to $\mathbf{R}, \mathrm{b}=3.05, \mathrm{t}(108)=8.22, \mathrm{p}<0.001$, and compared to $\mathbf{V}, \mathrm{b}=1.67, \mathrm{t}(108)=4.52, \mathrm{p}<0.001$. There was no significant difference in direction error between $\mathbf{T}$ and $\mathbf{V}+\mathbf{T}, \mathrm{b}$ $=0.34, t(108)=0.93, p=0.35$. In order to compare the significant pairwise differences, we produced a post-hoc test as set out below (see Fig. 10):

- Compared to $\mathbf{R}$, direction error was significantly reduced for $\mathbf{T}(\mathrm{p}<0.001), \mathbf{V}+\mathbf{T}(\mathrm{p}<0.001)$ and $\mathbf{V}(\mathrm{p}=0.001)$.

- Compared to $\mathbf{V}$, direction error was significantly reduced for $\mathbf{T}(\mathrm{p}<0.001)$ and $\mathbf{V}+\mathbf{T}(\mathrm{p}=0.0017)$ representations.

- There was no significant difference in direction error using $\mathbf{V}+\mathbf{T}$ compared to $\mathbf{T}(\mathrm{p}=0.78)$.

\subsection{Wind Force}

The experimental condition had a significant effect on the estimation of wind force, $\chi^{2}(3)=13.54, p=0.0036$. Orthogonal contrast revealed that force error (difference between the expected response and the value given by the participants) was significantly reduced for $\mathbf{T}$ compared to $\mathbf{R}, \mathrm{b}=0.18, \mathrm{t}(108)=2.64, \mathrm{p}=0.0094$. There was not significant difference in force error in $\mathbf{T}$ compared to $\mathbf{V}, \mathrm{b}=$ $0.14, t(108)=1.99, p=0.05$, and to $\mathbf{V}+\mathbf{T}, \mathrm{b}=-0.039, \mathrm{t}(108)=-0.56$, $\mathrm{p}=0.57$. In order to compare the significant pairwise differences, we produced a post-hoc test as set out below (see Fig. 11):

- Compared to the $\mathbf{R}$, force error was significantly reduced for $\mathbf{T}(\mathrm{p}=0.039)$ and $\mathbf{V}+\mathbf{T}(\mathrm{p}=0.0068)$ 


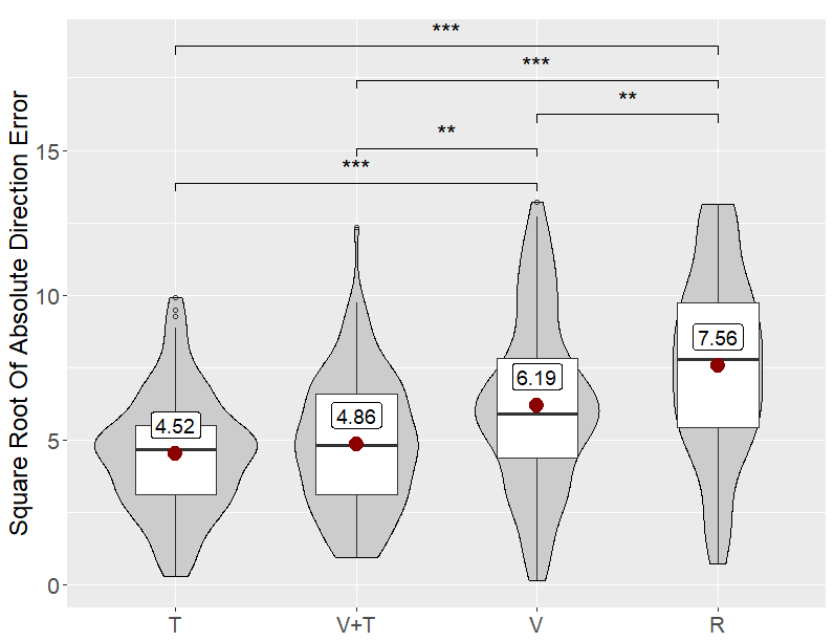

Figure 10: Box plot and violin plot of the square root of the wind direction estimation error through the four different representations. The red dot is the mean value. The horizontal lines represent the quartiles. The violin plot shows the frequency of results. For each plot, the stars present the level of significance $(*=p<0.05, * *=p$ $<0.01, * * *=\mathrm{p}<0.001)$.

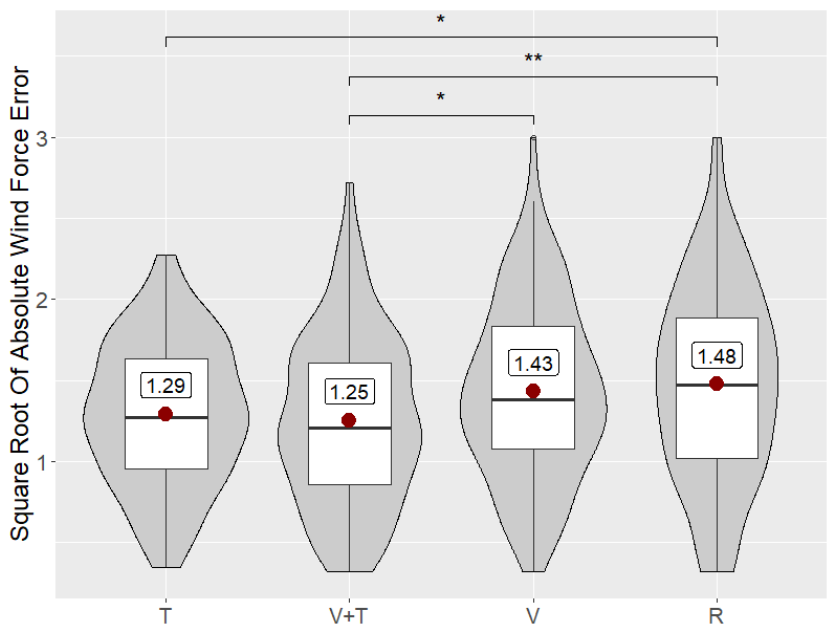

Figure 11: Box plot and violin plot of the square root of the absolute wind force error through the four different representations.

- Compared to the $\mathbf{V}$, force error was significantly reduced for $\mathbf{V}+\mathbf{T}(\mathrm{p}=0.049)$.

- Compared to $\mathbf{V}$, the wind force error was not significantly different either with $\mathbf{R}(\mathrm{p}=0.91)$ nor $\mathbf{T}(\mathrm{p}=0.18)$.

- There was no significant difference in force error for $\mathbf{T}$ compared to $\mathbf{V}+\mathbf{T}(\mathrm{p}=0.94)$.

\subsection{Sense of Presence}

The experimental condition also had a significant effect on the estimation of the sense of presence, $\chi^{2}(3)=53.543, p<0.001$. Orthogonal contrast revealed that the sense of presence was significantly increased in $\mathbf{T}$ compared to $\mathbf{R}, \mathrm{b}=-0.17, \mathrm{t}(108)=-5.60, \mathrm{p}<0.001$, $\mathbf{V}, \mathrm{b}=-0.24, \mathrm{t}(108)=-7.66, \mathrm{p}<0.001$ and $\mathbf{V}+\mathbf{T}, \mathrm{b}=-0.08, \mathrm{t}(108)=$

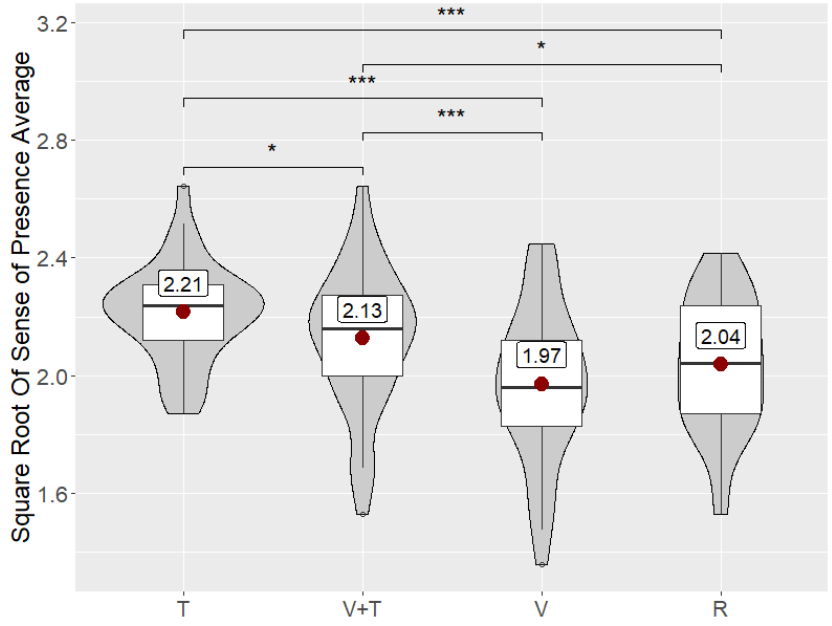

Figure 12: Box plot and violin plot of the square root of the average sense of presence through the four different representations. The higher the value, the higher the sense of presence.

$-2.78, p=0.006$. In order to compare the significant pairwise differences, we produced a post-hoc test as set out below (see Fig. 12):

- Sense of presence was significantly increased for $\mathbf{T}$ compared to $\mathbf{R}(\mathrm{p}<0.001), \mathbf{V}(\mathrm{p}<0.001)$ and $\mathbf{V}+\mathbf{T}(\mathrm{p}=0.024)$.

- Sense of presence was significantly increased for $\mathbf{V + T}$ compared to $\mathbf{R}(\mathrm{p}=0.023)$ and compared to $\mathbf{V}(\mathrm{p}<0.001)$.

- There was no significant difference in the sense of presence for $\mathbf{V}$ compared to $\mathbf{R}(\mathrm{p}=0.15)$.

\section{Discussion}

The results of this study show that, according to conditions and types of representation used in our prototype, $\mathbf{T}$ condition is the most accurate with regard to D1 (wind direction) and is the one with the largest increase to $\mathbf{D 3}$ (sense of presence). $\mathbf{V}+\mathbf{T}$ was the most accurate with regard to $\mathbf{D 2}$ (wind force).

T enables a better assessment of the wind direction, thus rejecting H1. The streamlines used in the study for the conditions $\mathbf{V}$ and $\mathbf{V}+\mathbf{T}$ are, according to Laramee et al. [24], more suitable for the $3 \mathrm{D}$ visualizations than direct ones (because of occlusions, visual cluttering or lack of depth). Yet, there exist several ways of understanding the streamlines: They can be either considered as a whole thus indicating a global direction or they can be considered more locally and seen as some kind of vortex. This might explain why, even if $\mathbf{V}$ was more accurate than $\mathbf{R}$, it was not as good as $\mathbf{T}$.

$\mathbf{V}+\mathbf{T}$ results do not support $\mathbf{H 2}$. When adding the tactile restitution to the visual effects, the wind direction perception is improved with respect to $\mathbf{V}$ but is not significantly different from $\mathbf{T}$. It shows that, when evaluating the wind direction, the tactile effect was dominant over the visual one.

While the mean estimate of the wind force tends to be more accurate for $\mathbf{T}$ than $\mathbf{V}$ value, $\mathbf{H 3}$ cannot be formally accepted. In contrast to $\mathbf{V}, \mathbf{T}$ and $\mathbf{V}+\mathbf{T}$ conditions were significantly different from $\mathbf{R}$. During the $\mathbf{V}+\mathbf{T}$ condition, the participants tended to be more accurate in their perception of the wind force. However this condition was not significantly different from $\mathbf{T}$, thus not allowing to accept $\mathbf{H 4}$.

With reference to the sense of presence in the VR scene, results show that $\mathbf{T}$ was the condition that increased it most, supporting 
H5 and H6. The $\mathbf{V}$ condition decreased the sense of presence with regard to $\mathbf{R}$. This can be explained by the fact that we made visible something that is not visible in the real world. When adding tactile effects to visual effects $(\mathbf{V}+\mathbf{T})$, the sense of presence was increased compared to $\mathbf{R}$. Even if $\mathbf{V}+\mathbf{T}$ is less than $\mathbf{T}$, this study could be broadened with different visual stimuli. Compared to $\mathbf{R}$ condition, $\mathbf{T}$ was better, this is consistent with previous similar studies [21,25].

\section{Limitations AND FUtURE WORK}

Comparing the wind properties with regard to its visual and tactile effects was one of the main objectives of this study. To do this, we used a streamlines representation that allows us to trace the wind flow along its path. This visualization then allows us to identify the interaction between urban shapes and the wind. However, due to the 3D nature of streamlines, it can be confusing when trying to understand the wind direction, which is evaluated in two dimensions. Streamlines could bring a better idea of the global flow direction, but could prove more difficult to identify the specific wind direction in a specific place of the space, i.e. the exact moment where the line impacts the user.

In traditional information visualization, the wind force is usually represented by a color degradation in a static image. In our prototype, it is visually represented by the speed of a $3 \mathrm{D}$ object. This may limit the perception of small speed changes and could explain the similarity between $\mathbf{R}$ and $\mathbf{V}$. This is why we believe studying other kinds of visual wind flow effects could be more appropriate for future analyses.

Some limitations also arise from the representation and the measurement of wind characteristics. Our 8 fans had only 4 possible speeds each thus creating, even combined, a limited number of possible wind force and direction couples. On the measurement side, we used a 1 to 10 scale that had no physical equivalent and consequently does not allow us to fully measure and compare to the real wind speed. Note that this effect is present in all the conditions. The estimation of wind properties in a real situation can be complicated without the necessary measurement tools (traditional wind estimations methods are reference-based, e.g the Beaufort scale [13] describes the mechanical effects of the wind on the environment).

Finally, we would also like to stress that wind simulation, as any result from CFD computation is also very sensitive to mesh size and initial data and would require a validation process by comparing to real data. The goal of this paper was more to assess the perception of simulation results than to assess, at this stage, an accurate simulation of a real street. That would however constitute an interesting matter for collaboration between simulation and final users such as architects. Ultimately, an AR-based visualisation could be of interest as well.

Another potential limitation of this experiment is the use of a large unique screen instead of an HMD. While the former has the advantage of not covering the face for tactile stimulation, the latter could provide a better immersion thanks to a larger field of view and by making the fans totally invisible. In our next experiment, we plan a comparison which should provide interesting results. At this stage, our study is based on qualitative wind perception, we also would like to assess the JNDs reachable with our system as described by Nakano et al. [30].

\section{Conclusion}

In this paper, we presented a user study aiming at comparing the perception and understanding of wind properties (direction and force) and their influence on the sense of presence in a VR scene. It was carried out under four different conditions: (R) audiovisual representation of the mechanical effects of the wind on the context elements, (V) visual representation of the wind flow, (T) tactile restitution of the wind with the help of digitally controlled floor fans and the $(\mathrm{V}+\mathrm{T})$ condition combining $(\mathrm{V})$ and $(\mathrm{T})$.
Results show a better understanding of the wind direction, the wind force, and the sense of presence with conditions including tactile restitution. For the wind direction and sense of presence, only the addition of tactile wind (coming from the fans) to the reference scene improves the accuracy. In terms of wind force, the tactile and visual effects added to the reference scene enables the best assessment.

This study shows that there may be an interest in the architecture and urban design fields to exploit other types of representations, beyond the visualization of wind flow, to introduce wind behavior at different stages of a project. The tactile wind restitution around the project representation could help to simulate or reproduce a possible wind behavior from an urban space. The results show the proper understanding of the suggestion of wind direction and force expressed from a CFD simulation using a tactile restitution. These results, added to the increased sense of presence using the tactile restitution, show an interest in the architectural field to simulate the resulting conditions of the interaction between the urban morphology and the environmental phenomena.

\section{ACKNOWLEDGMENTS}

The authors wish to thanks Houda Belgacem, Pierre Molinaro, Léa Pillette, Sophie Provost, Maxim Spur, Jenny Gutiérrez and Daniel Siret for their help in setting up the system and proofreading the paper.

\section{REFERENCES}

[1] B. Bakay and W. Heidrich. Real-Time Animated Grass. In Proceedings of Eurographics (short paper), 2002.

[2] M. Barr. Late Rain - Waymouth Street, 2016. Library Catalog: fineartamerica.com. Available at https://fineartamerica.com/ featured/late-rain-waymouth-street-mike-barr.html.

[3] M. Berger and V. Cristie. CFD post-processing in Unity3D. Procedia Computer Science, 51:2913-2922, Dec. 2015. doi: 10.1016/j.procs. 2015.05.476

[4] B. Blocken, T. Stathopoulos, J. Cameliet, and J. Hensen. Application of CFD in building performance simulation for the outdoor environment. IBPSA, 2009.

[5] S. Botticelli. The birth of venus, 1485.

[6] A. Brambilla, R. Carnecky, R. Peikert, I. Viola, and H. Hauser. Illustrative Flow Visualization: State of the Art, Trends and Challenges. pp. 75-94, Jan. 2012. doi: 10.2312/conf/EG2012/stars/075-094

[7] C. Bustamante, M. Jans, and E. Higueras. The behavior of wind in urban morphology and its incidence in the resting use of public space, Punta Arenas, Chile, (in spanish). AUS, (15):28-33, 2014. doi: 10. 4206/aus.2014.n15-06

[8] S. Cardin, D. Thalmann, and F. Vexo. Head Mounted Wind. proceeding of the 20th annual conference on Computer Animation and Social Agents (CASA2007), pp. 101-108, 2007.

[9] J. Diener, M. Rodriguez, L. Baboud, and L. Reveret. Wind projection basis for real-time animation of trees. Computer Graphics Forum, 28(2):533-540, Mar. 2009. doi: 10.1111/j.1467-8659.2009.01393.x

[10] Y. Dobashi, K. Kaneda, H. Yamashita, T. Okita, and T. Nishita. A Simple, Efficient Method for Realistic Animation of Clouds. In Proceedings of the 27th Annual Conference on Computer Graphics and Interactive Techniques, SIGGRAPH '00, pp. 19-28. ACM Press/AddisonWesley Publishing Co., New York, NY, USA, 2000. doi: 10.1145/ 344779.344795

[11] M. Edmunds, R. S. Laramee, G. Chen, N. Max, E. Zhang, and C. Ware. Surface-based flow visualization. Computers \& Graphics, 36(8):974 990, 2012. Graphics Interaction Virtual Environments and Applications 2012. doi: 10.1016/j.cag.2012.07.006

[12] A. P. Field, J. Miles, and Z. Field. Discovering statistics using R / Andy Field, Jeremy Miles, Zoë Field. London ; Thousand Oaks, Calif. : Sage, 2012.

[13] W. M. O. C. for Maritime Meteorology. The Beaufort Scale of Wind Force : (Technical and Operational Aspects). Geneva :WMO, 1970. 
[14] M. Gabriel and E. Goldberg. Pocahontas, Nov. 1995. IMDb ID: $\mathrm{tt} 0114148$

[15] J. Gandemer. Discomfort due to wind near buildings: aerodynamic concepts. NBS technical note;710-9. Dept. of Commerce, National Bureau of Standards : For sale by the Supt. of Docs., U.S. Govt. Print. Off., Washington, 1978.

[16] G. Giraldo, M. Servières, V. Tourre, V. Signorelli, and A. Bonnet. Caractérisation et restitution vidéographique des ambiances urbaines. In SCAN'18, vol. 47. SHS Web of Conferences, Nantes, 2018. doi: 10. $1051 /$ shsconf $/ 20184701012$

[17] E. T. Hall. The hidden dimension. Garden City, N.Y: Anchor Books, 1969.

[18] K. Ito, Y. Ban, and S. Warisawa. Alteredwind: Manipulating perceived direction of the wind by cross-modal presentation of visual, audio and wind stimuli. In SIGGRAPH Asia 2019 Emerging Technologies, SA '19, p. 3-4. Association for Computing Machinery, New York, NY, USA, 2019. doi: 10.1145/3355049.3360525

[19] J. Kahrs. Paperman, Dec. 2012. IMDb ID: tt2388725.

[20] Y. Kojima, Y. Hashimoto, and H. Kajimoto. A Novel Wearable Device to Present Localized Sensation of Wind. In Proceedings of the International Conference on Advances in Computer Enterntainment Technology, ACE '09, pp. 61-65. ACM, New York, NY, USA, 2009. doi: $10.1145 / 1690388.1690399$

[21] S. Kulkarni, M. Minor, M. Deaver, E. Pardyjak, and J. M. Hollerbach. Design, Sensing, and Control of a Scaled Wind Tunnel for Atmospheric Display. Mechatronics, IEEE/ASME Transactions on, 17:1-11, Aug. 2012. doi: 10.1109/TMECH.2011.2113353

[22] R. Lamberts, L. Dutra, and F. O. R. Pereira. Eficiência Energética na Arquitetura. ELETROBRAS/PROCEL, Rio de Janeiro, $3^{\text {a }}$ edição ed., 2014.

[23] R. S. Laramee, D. Weiskopf, J. Schneider, and H. Hauser. Investigating Swirl and Tumble Flow with a Comparison of Visualization Techniques. In In Proceedings Ieee Visualization '04, pp. 51-58. IEEE Computer Society, 2004.

[24] R. S. Laramee, D. Weiskopf, J. Schneider, and H. Hauser. Investigating swirl and tumble flow with a comparison of visualization techniques. In IEEE Visualization 2004, pp. 51-58, Oct. 2004. doi: 10.1109/VISUAL .2004 .59

[25] A. Lehmann, C. Geiger, B. Woldecke, and J. Stocklein. Poster: Design and evaluation of 3D content with wind output. In 2009 IEEE Symposium on 3D User Interfaces, pp. 151-152, Mar. 2009. doi: 10. 1109/3DUI.2009.4811231

[26] N. Max, R. Crawfis, and D. Williams. Visualizing Wind Velocities by Advecting Cloud Textures. In Proceedings of the 3rd Conference on Visualization '92, VIS '92, pp. 179-184. IEEE Computer Society Press, Los Alamitos, CA, USA, 1992.

[27] T. McLoughlin, R. S. Laramee, R. Peikert, F. H. Post, and M. Chen Over two decades of integration-based, geometric flow visualization. Computer Graphics Forum, 29(6):1807-1829, 2010. doi: 10.1111/j. 1467-8659.2010.01650.x

[28] T. Moon and G. J. Kim. Design and Evaluation of a Wind Display for Virtual Reality. In Proceedings of the ACM Symposium on Virtual Reality Software and Technology, VRST '04, pp. 122-128. ACM, New York, NY, USA, 2004. doi: 10.1145/1077534.1077558

[29] T. Nakano, S. Saji, and Y. Yanagida. Indicating wind direction using a fan-based wind display. In P. Isokoski and J. Springare, eds., Hap tics: Perception, Devices, Mobility, and Communication, pp. 97-102. Springer Berlin Heidelberg, Berlin, Heidelberg, 2012.

[30] T. Nakano, Y. Yoshioka, and Y. Yanagida. Effects of wind source configuration of wind displays on property of wind direction perception. In ACHI 2014 : The Seventh International Conference on Advances in Computer-Human Interactions, 2014.

[31] D. Paes, E. Arantes, and J. Irizarry. Immersive environment for improving the understanding of architectural 3D models: Comparing user spatial perception between immersive and traditional virtual reality systems. Automation in Construction, 84:292-303, Dec. 2017. doi: 10. 1016/j. autcon.2017.09.016

[32] J. Park, Y. Jin, S. Ahn, and S. Lee. The impact of design representation on visual perception: Comparing eye-tracking data of architectural scenes between photography and line drawing. Archives of Design
Research, 32(1):5-29, Jan. 2019. Publisher: Korean Society of Design Science. doi: 10.15187/ADR.2019.02.32.1.5

[33] J. P. Pluijms, R. Cañal-Bruland, W. M. Bergmann Tiest, F. A. Mulder, and G. J. P. Savelsbergh. Expertise effects in cutaneous wind perception. Attention, Perception \& Psychophysics, 77(6):2121-2133, 2015. doi: 10.3758/s13414-015-0893-6

[34] F. H. Post, B. Vrolijk, H. Hauser, R. S. Laramee, and H. Doleisch. The State of the Art in Flow Visualisation: Feature Extraction and Tracking. Computer Graphics Forum, 22(4):775-792, Feb. 2004. doi: 10.1111/j. 1467-8659.2003.00723.x

[35] E. Quigley, Y. Yu, J. Huang, W. Lin, and R. Fedkiw. Real-Time Interactive Tree Animation. IEEE Transactions on Visualization and Computer Graphics, 24(5):1717-1727, May 2018. doi: 10.1109/TVCG 2017.2661308

[36] M. Rietzler, K. Plaumann, T. Kränzle, M. Erath, A. Stahl, and E. Rukzio. VaiR: Simulating 3D Airflows in Virtual Reality. In Proceedings of the 2017 CHI Conference on Human Factors in Computing Systems, CHI '17, pp. 5669-5677. ACM, New York, NY, USA, 2017. doi: $10.1145 / 3025453.3026009$

[37] T. Salzbrunn, H. Janicke, T. Wischgoll, and G. Scheuermann. The State of the Art in Flow Visualization: Partition-Based Techniques. Proceedings of the 2008 Simulation and Visualization Conference, pp. 75-92, Feb. 2008.

[38] M. N. Shyamalan. The happening, June 2008. IMDb ID: tt0949731.

[39] M. Slater, J. McCarthy, and F. Maringelli. The Influence of Body Movement on Subjective Presence in Virtual Environments. Human Factors, 40(3):469-477, Sept. 1998. doi: 10.1518/001872098779591368

[40] S. Tahrani and G. Moreau. Integration of Immersive Walking to Analyse Urban Daylighting Ambiences. Journal of Urban Design, 13(1):99-123, Feb. 2008. Publisher: Routledge _eprint: https://doi.org/10.1080/13574800701803506. doi: 10.1080/ 13574800701803506

[41] S. Unseld. The Blue Umbrella, July 2013. IMDb ID: tt2616880.

[42] J. C. Verlinden, F. A. Mulder, J. S. Vergeest, A. de Jonge, D. Krutiy, Z. Nagy, B. J. Logeman, and P. Schouten. Enhancement of Presence in a Virtual Sailing Environment through Localized Wind Simulation. Procedia Engineering, 60:435-441, Jan. 2013. doi: 10.1016/j.proeng. 2013.07.050

[43] T. Vigier, D. Siret, G. Moreau, and L. Lescop. Sensitive suggestion and perception of climatic effects in virtual urban environments. ACM Symposium on Applied Perception, 2013.

[44] C. Wang, Z. Wang, T. Xia, and Q. Peng. Real-time snowing simulation. The Visual Computer, 22(5):315-323, May 2006. doi: 10.1007/s00371 $-006-0012-8$ 